\title{
FIXED POINTS UNDER TRANSFORMATIONS OF CONTINUA WHICH ARE NOT CONNECTED IM KLEINEN*
}

\author{
BY \\ O. H. HAMILTON
}

1. Introduction. Ayres $\dagger$ has shown that if $M$ is a compact continuous curve in the plane which does not separate the plane and $T$ is a reversibly continuous transformation of $M$ into a subset of itself, then $T$ leaves some point of $M$ invariant. He also proves similar theorems for special cases of non-planar continuous curves. The purpose of this paper is to extend these results to certain types of continua which are not connected im kleinen.

2. Preliminary theorem and lemmas. We prove first the following preliminary theorems and lemmas.

Theorem I. If $M$ is a compact continuum in a metric space but is not an indecomposable continuum, and if $T$ is any reversibly continuous transformation of $M$ into a subset of itself, then some proper subcontinuum $N$ of $M$ contains $a$ point of $T(N)$, the image of $N$ under $T$.

Proof. If $T$ carries $M$ into a proper subset of itself, then $T(M)$ contains its image $T^{2}(M)$ and the theorem is true. Suppose then that $T$ carries $M$ into itself. It can easily be shown that if $M$ is not indecomposable, it does not contain two mutually exclusive composants. The transformation $T$ carries a composant of $M$ into a composant of $M$. It has been shown that a composant $\ddagger$ of a continuum is the sum of a countable number of continua $N_{1}, N_{2}, N_{3}, \cdots$, where for each integer $i, N_{i}$ contains $N_{i-1}$.

Let $V$ be any composant of $M$, and let $T(V)$ be its image under $T$. Then $V$ and $T(V)$, by what was said above, must have a point in common. Let $V$ be expressed as $\sum_{i=1}^{\infty} V_{i}$, where for each integer $i, V_{i}$ is a continuum which contains $V_{i-1}$. Then $T(V)=\sum_{i=1}^{\infty} T\left(V_{i}\right)$ where for each integer $i, T\left(V_{i}\right)$ is the image of $V_{i}$ under $T$ and is a continuum which contains $T\left(V_{i-1}\right)$. For some integer $j, V_{j}$ contains a point of $T\left(V_{j}\right)$, for if we suppose the contrary to be true, it is obvious that $V$ contains no point of $T(V)$, contradicting the fact

* Presented to the Society, September 10, 1937; received by the editors May 29, 1937.

† W. L. Ayres, Some generalizations of the Scherrer fixed-point thecrem, Fundamenta Mathematicae, vol. 16 (1930), pp. 333-336.

† See R. L. Moore, Foundations of Point Set Theory, American Mathematical Society Colloquium Publications, vol. 13, p. 75. 
that $V$ and $T(V)$ have a point in common. $V_{j}$ is therefore a continuum satisfying the conclusions of the theorem.

LEMMA 1. If $M$ is a compact continuum in a metric space and $M$ is not an indecomposable continuum and contains no continuum which is the sum of two continua whose common part is disconnected, and if $T$ is a reversibly continuous transformation of $M$ into a subset of itself and neither $T$ nor $T^{-1}$ carries a proper subcontinuum of $M$ into itself, then there is a proper subcontinuum $L_{0}$ of $M$ such that $L_{0}$ contains a point of $T\left(L_{0}\right)$ but such that for $n>1$, the common part of $L_{0}$ and $T^{n}\left(L_{0}\right)$ is vacuous.

Proof. By Theorem I, some proper subcontinuum $K_{0}$ of $M$ contains a point of its image $T\left(K_{0}\right)$. For each positive or negative integer $n$, let $K_{n}$ designate $T^{n}\left(K_{0}\right)$. By a hypothesis of the theorem, $K_{0}$ is not identical with $K_{1}$, for suppose $K_{0}=K_{1}$. Then $K_{0}$ is a proper subcontinuum of $M$ which is carried into itself by $T$. Furthermore $K_{0} \cdot K_{1}$ is not identical with $K_{0}$ or with $K_{1}$; for suppose $K_{0} \cdot K_{1}$ is identical with $K_{0}$. Then $T$ carries $K_{0}$ into a subset of itself. Consider the continuum $A=\prod_{n=0}^{\infty} K_{n}$. Then $T(A)=A$, and $T$ carries $A$, a proper subcontinuum of $M$, into itself. Similarly, if $K_{0} \cdot K_{1}$ is identical with $K_{1}$, $T^{-1}$ carries a proper subcontinuum of $M$ into itself; thus we have a contradiction of a hypothesis of the theorem.

There exists an integer $r$ such that the $\prod_{i=0}^{r} K_{i}$ is not vacuous but such that $\prod_{i=0}^{r+1} K_{i}$ is vacuous. This can be shown as follows. $K_{0} \cdot K_{1}$ is not vacuous. Suppose $\prod_{i=0}^{s} K_{i}$ contains a point for each integer $s$. Then $B=\prod_{n=0}^{\infty} K_{n}$ is not vacuous and is a proper subcontinuum of $M$ which is carried into itself by $T$, which is, again, a contradiction of a hypothesis of the theorem. Let $L_{0}$ be the continuum $\prod_{i=0}^{r-1} K_{i}$, where $r$ is an integer such that $\prod_{i=0}^{r} K_{i}$ is not vacuous but $\prod_{i=0}^{r+1} K_{i}$ is vacuous. For each integer $i$, let $L_{i}=T^{i}\left(L_{0}\right)$. Then $L_{1}$ is $\prod_{i-1}^{r} K_{i}$. $L_{0} \cdot L_{1}$ is not vacuous since $L_{0} \cdot L_{1}=\prod_{i=0}^{r} K_{i}$ which is not vacuous. $L_{0} \cdot L_{2}$ is vacuous. For suppose $L_{0}$ contains a point of $L_{2}$. Then $\prod_{i=0}^{r-1} K_{i}$ contains a point $P$ of $\prod_{i=2}^{r+1} K_{i}$. It follows that $P$ is in $\prod_{i=0}^{r+1} K_{i}$, which by hypothesis is vacuous. Furthermore $L_{0}$ contains no point of $L_{r}, r>2$. For suppose the contrary and that $s$ is the smallest integer greater than two such that $L_{0}$ contains a point of $L_{s}$. Consider the two continua, $L_{0}+L_{s}$ and $L_{1}+L_{2}+\cdots+L_{s-1}$. Their common part is the sum of the two mutually exclusive continua, $L_{0} \cdot L_{1}$ and $L_{s-1} \cdot L_{s}$. Then $\sum_{i=0}^{s} L_{i}$ is a subcontinuum of $M$ which is the sum of two continua whose common part is disconnected. This is a contradiction of a hypothesis of the theorem. The lemma is therefore true.

LEMMA 2. If, in a metric space, $M$ is a compact non-degenerate continuum but is not an indecomposable continuum and does not contain a continuum which is the sum of two continua whose common part is disconnected, and if $T$ is 
a reversibly continuous transformation of $M$ into a subset of itself, then $T$ carries some proper subcontinuum of $M$ into itself.

Proof. Suppose $T$ does not carry a proper subcontinuum of $M$ into itself. Then by Lemma 1 , there exists a sequence of subcontinua $L_{0}, L_{1}, L_{2}, \ldots$ such that (1) for each positive and negative integer $n, L_{n}$ is $T^{n}\left(L_{0}\right),(2) L_{n}$ and $L_{n \pm 1}$ have a point in common, but $L_{n}$ and $L_{n+r},|r|>1$, do not have a point in common. Let $V_{0}$ be an irreducible continuum from $L_{-1} \cdot L_{0}$ to $L_{0} \cdot L_{1}$ which is a subcontinuum of $L_{0}$. Let $V_{n}$, for each positive or negative integer, designate $T^{n}\left(V_{0}\right)$. Then since $T$ is reversibly continuous, it can be easily shown that $V_{n}$ is irreducible from $L_{n-1} \cdot L_{n}$ to $L_{n} \cdot L_{n+1}$. Let $P_{0}$ be a point of $V_{0}$ not in $L_{-1} \cdot L_{0}$ or $L_{0} \cdot L_{1}$. There is such a point for otherwise $L_{-1}$ would contain a point of $L_{1}$. For each positive or negative integer $n$, let $P_{n}$ designate $T^{n}\left(P_{0}\right)$. Let $M_{0}$ be an irreducible continuum from $P_{0}$ to $P_{1}$ which is a subset of $V_{0}+V_{1}+L_{0} \cdot L_{1}$. Then since $T$ is reversibly continuous, it follows that (1) $M_{n}$ for each integer $n$ is irreducible from $P_{n}$ to $P_{n+1}$, (2) $M_{n} \cdot M_{n \pm 1}$ is not vacuous, but $M_{n} \cdot M_{n+r},|r|>1$, is vacuous, (3) $\left(M_{i}+M_{i-1}\right) \cdot V_{i}$ is an irreducible continuum from $L_{i-1} \cdot L_{i}$ to $L_{i} \cdot L_{i+1}$.

Since $M$ is not indecomposable, $M$ is the sum of two proper subcontinua $H$ and $K$. One of these continua contains points of infinitely many of the continua $M_{0}, M_{1}, M_{2}, \cdots$. Suppose $H$ contains points of infinitely many of these continua. Let $j$ be the smallest integer greater than or equal to zero such that $H$ contains a point of $M_{j}$. Let $r$ be any integer greater than 5 such that $H$ contains a point of $M_{j+r}$. Let $H^{\prime}$ designate the common part of $H$ and the continuum $\sum_{i=j}^{j+r} M_{i}$. But $H^{\prime}$ is itself a continuum since the common part of two subcontinua of $M$ cannot be disconnected. $H^{\prime}$ must contain points of $L_{j+1} \cdot L_{j+2} \cdot M_{j+1}, L_{j+2} \cdot L_{j+3} \cdot M_{j+2}, \cdots, L_{j+r-2} \cdot L_{j+r-1} \cdot M_{j+r-2}$, since each of these continua separates $M_{j}$ from $M_{j+r}$ in $\sum_{i=j}^{++r} M_{i}$. It follows that $H^{\prime}$ contains $P_{j+2}, P_{j+3}, \cdots, P_{j+r-2}$, since for each integer $s, P_{j+s}$ lies in a subcontinuum of $\sum_{i=j}^{j+r+1} L_{i}$ which is irreducible from $L_{j+s-1} \cdot L_{j+s}$ to $L_{j+s} \cdot L_{j+s+1}$. Then $H^{\prime}$ contains each of the continua $M_{j+2}, M_{j+3}, \cdots, M_{j+r-3}$; since each of these continua is irreducible between two points of $\sum_{i=j+1}^{j+r-2} P_{i}$. Since $r$ can be taken arbitrarily large, it follows that $H^{\prime}$ contains $M_{j+n}$ for each integer $n$ greater than 1.

Consider the continuum $C=\overline{\sum_{i=j+2}^{\infty} M_{i}}$. It is obvious that $T(C)$ is a subset of $C$. It follows that $C$ is identical with $M$, for otherwise $C$ contains a proper subcontinuum of $M$ which is carried into itself by $T$, contrary to hypothesis. But by the argument given above $C$ is a subset of $H$; therefore $H$ is identical with $M$. This contradicts the assumption that $H$ is a proper subcontinuum of $M$. The lemma is therefore true. 
3. A general theorem on fixed points. We may now prove the general theorem:

TheOREM II. If $M$ is a compact continuum in a metric space, and if $M$ does not contain an indecomposable continuum and does not contain a continuum which is the sum of two continua whose common part is disconnected, then every reversibly continuous transformation of $M$ into a subset of itself leaves some point of $M$ invariant.

Proof. By Lemma 2, $T$ carries some proper subcontinuum $M_{1}$ of $M$ into itself. Let $M_{1}, M_{2}, \cdots, M_{\omega}, M_{\omega+1}, \cdots$ be a well ordered sequence $\beta$ of subcontinua of $M$ having the following properties: (1) for each ordinal number $\theta$ which has an immediate predecessor, $M_{\theta}$ is a proper subcontinuum of $M_{\theta-1}$ which is carried into itself by $T$; $(2)$ for each ordinal number $\phi$ which has no immediate predecessor, $M_{\phi}$ is a proper subcontinuum of $M_{\alpha}$ which is carried into itself by $T$, where $\alpha$ is any ordinal which is less than $\phi$; (3) if $M_{\lambda}$ is any non-degenerate continuum which belongs to the sequence $\beta, M_{\lambda+1}$ is a proper subcontinuum of $M_{\lambda}$ belonging to the sequence $\beta$. Since $M$ is metric, it is completely separable, and it follows that the sequence $\beta$ thus defined is countable. Therefore there exists a countable simple subsequence $M_{n 1}, M_{n 2}, \ldots$ running through $\beta$. That is, if $M_{\lambda}$ is any element of $\beta$, there is an integer $i$ such that $M_{n i}$ is a subcontinuum of $M_{\lambda}$. Since $M$ is compact, the point set $P=\prod_{i=1}^{\infty} M_{n i}$ is a compact continuum and is carried into itself by $T$, since each of the continua $M_{n i}$ is carried into itself by $T$. But $P$ cannot be a nondegenerate continuum, for if it is, it has a proper subcontinuum which belongs to the sequence $\beta$; and this contradicts the definitions of $P$ and of the sequences $\beta$ and $M_{n i}$. It follows that $P$ is a point of $M$, invariant under $T$.

4. Applications to continua in the plane. We have now the following applications:

THEOREM III. If $M$ is a compact continuum in the plane which contains no indecomposable continuum, which does not separate the plane, and which contains no domain, then every reversibly continuous transformation of $M$ into a subset of itself leaves some point invariant.

Proof. It follows from a theorem proved by S. Janiszewski* and also by Miss Mullikan $\dagger$ that a sufficient condition that a compact continuum $M$ separate the plane is that $M$ be the sum of two continua whose common

* S. Janiszewski, Sur les coupures du plan faites par les continus, Prace Matematyczno-fizyczne, vol. 26 (1913), pp. 11-63.

† Anna M. Mullikan, Certain theorems relating to plane connected point sets, these Transactions, vol. 24 (1922), pp. 144-162. 
part is disconnected. Then if $M$ does not separate the plane and contains no domain, no subcontinuum of $M$ separates the plane, and the hypotheses of Theorem II are satisfied. The conclusions of Theorem III then follow.

THEOREM IV. If $D$ is a bounded simply connected domain in the plane which, together with its boundary, does not separate the plane and whose outer boundary $M$ contains no indecomposable continuum, then every reversibly continuous transformation of $D$ into itself leaves some point of $D$ invariant.

Proof. Without loss of generality we may suppose the boundary of $D$ to be identical with its outer boundary, for the compact complementary domain of the outer boundary $M$ of $D$ is itself a simply connected domain which is a subset of $D$ and whose boundary is $M$. Carathéodory* has shown that there exists a conformal and reversibly continuous transformation $T_{1}$ of $D$ into the interior $I$ of a given circle $J$, that there exists a reversibly one-to-one correspondence, also designated by $T_{1}$, between the prime ends of $D$ and the points of $J$, and that this correspondence is characterized as follows: If $P_{1}, P_{2}, P_{3}, \cdots$ is a sequence of points of $D$ converging to a prime end $E_{P}$ of $D$ (using Carathéodory's definition of convergence), then the sequence of points $T_{1}\left(P_{1}\right), T_{1}\left(P_{2}\right), T_{1}\left(P_{3}\right), \cdots$ converges (in the usual sense) to the point $P$ of $J$ with which $E_{P}$ is associated by this correspondence; and conversely, if $Q_{1}, Q_{2}, Q_{3}, \cdots$ is a sequence of points of $I$ converging to a point $Q$ of $J$, then the sequence of points $T_{1}^{-1}\left(Q_{1}\right), T_{1}^{-1}\left(Q_{2}\right), T_{1}^{-1}\left(Q_{3}\right), \cdots$ converges to the prime end $E_{Q}$ with which $Q$ is associated by the correspondence $T_{1}$. Let $T$ be any reversibly continuous transformation of $D$ into itself. It can be easily shown that $T$ carries a prime end of $D$ into a prime end of $D$. Let $T_{2}$ be a transformation of the continuum $J+I$ into itself defined as follows: If $P$ is any point of $I$, let $T_{2}(P)$ be the point $T_{1}\left[T\left(T_{1}^{-1}(P)\right)\right]$. If $P$ is any point of $J$, let $P_{1}, P_{2}, \cdots$ be a sequence of points of $I$ converging to $P$. Let $E_{P}$ be the prime end of $D$ associated with $P$ by $T_{1}$. Then the sequence $T_{1}^{-1}\left(P_{1}\right), T_{1}^{-1}\left(P_{2}\right)$, $T_{1}^{-1}\left(P_{3}\right), \cdots$ converges to $E_{P}$. Since $T$ is reversibly continuous, the sequence $T\left(T_{1}^{-1}\left(P_{1}\right)\right), T\left(T_{1}^{-1}\left(P_{2}\right)\right), \cdots$ converges to the prime end $T\left(E_{P}\right)$ into which $E_{P}$ is transformed by $T$. It follows that the sequence of points of $I$, $T_{1}\left[T\left(T_{1}^{-1}\left(P_{1}\right)\right)\right], T_{1}\left[T\left(T_{1}^{-1}\left(P_{2}\right)\right)\right], \cdots$ converges to a point of $J$. That point is defined as $T_{2}(P)$. Since $T_{1}\left[T\left(T_{1}^{-1}\left(P_{i}\right)\right)\right]$ is defined as $T_{2}\left(P_{i}\right)$, and since $T$ is reversibly continuous on $D$, and $T_{1}$ is a reversible continuous transformation of $D$ into $I$, it follows that $T_{2}$ is reversibly continuous on $I$. Also from the definition of $T_{2}$ it follows that if $P_{1}, P_{2}, P_{3}, \cdots$ is a sequence of points of $I$ converging to a point $P$ of $J$, then the sequence $T_{2}\left(P_{1}\right), T_{2}\left(P_{2}\right), T_{2}\left(P_{3}\right), \ldots$

* C. Carathéodory, Über die Begrenzung einfach zusammenhängender Gebiete, Mathematische Annalen, vol. 73 (1912), pp. 323-370. 
converges to $T_{2}(P)$, and the sequence $T_{2}^{-1}\left(P_{1}\right), T_{2}^{-1}\left(P_{2}\right), T_{2}^{-1}\left(P_{3}\right), \cdots$ converges to $T_{2}^{-1}(P)$. Suppose that $Q_{1}, Q_{2}, Q_{3}, \cdots$ is a sequence of points of $J$ converging to a point $Q$ of $J$. For each positive integer $n$, let $Z_{n}$ be a point of $I$ such that each of the distances $d\left(T_{n}, Q_{n}\right)$ and $d\left[T_{2}\left(Z_{n}\right), T_{2}\left(Q_{n}\right)\right]$ is less than $1 / n$. That such a point $Z_{n}$ exists is shown as follows: There exists a sequence of points $Z_{n 1}, Z_{n 2}, Z_{n 3}, \cdots$ converging to $Q_{n}$. Then by the discussion above, the sequence $T_{2}\left(Z_{n 1}\right), T_{2}\left(Z_{n 2}\right), T_{2}\left(Z_{n 3}\right), \cdots$ converges to $T_{2}\left(Q_{n}\right)$. There exists an integer $i$ such that $d\left(Z_{n i}, Q_{n}\right)<1 / n$ and $d\left[T_{2}\left(Z_{n i}\right), T_{2}\left(Q_{n}\right)\right]<1 / n$. Then $Z_{n i}$ has the property required of $Z_{n}$. We see then that the convergence of the sequence $Q_{1}, Q_{2}, \ldots$ to $Q$ implies the convergence of the sequence $Z_{1}, Z_{2}, \ldots$ to $Q$. This in turn implies the convergence of $T_{2}\left(Z_{1}\right), T_{2}\left(Z_{2}\right), \cdots$ to $T_{2}(Q)$ and therefore the convergence of $T_{2}\left(Q_{1}\right), T_{2}\left(Q_{2}\right), \cdots$ to $T_{2}(Q)$. Similarly the sequence of points $T_{2}^{-1}\left(Q_{1}\right), T_{2}^{-1}\left(Q_{2}\right), \cdots$ converges to $T_{2}^{-1}(Q)$, and $T_{2}$ is a reversibly continuous transformation of the continuum $J+I$ into itself. By a fundamental theorem on fixed points, $T_{2}$ leaves some point of $J+I$ fixed. If $T_{2}$ leaves a point of $I$ fixed, then obviously $T$ leaves a point of $D$ fixed. If $T_{2}$ leaves a point $P$ of $J$ fixed, then $T$ carries some prime end $E_{P}$ of $D$ into itself. Let $N_{P}$ be the continuum in the boundary of $D$ associated with the prime end $E_{P}$ in the sense that every sequence of points of $D$ converging to $E_{P}$ has a subsequence which converges in the usual sense to a point of $N_{P}$. Then $T$ carries $N_{P}$ into itself. N. E. Rutt* has shown that a necessary condition that such a continuum as $N_{P}$ shall be the whole boundary of $D$ is that the boundary of $D$ shall be indecomposable or the sum of two indecomposable subcontinua. Since by hypothesis the boundary of $D$ contains no indecomposable continuum, $N_{P}$ is a proper subcontinuum of the boundary of $D . N_{P}$ then is a compact continuum which does not separate the plane and which contains no domain. It satisfies the hypothesis of Theorem III; therefore $T$ leaves some point of $N_{P}$ fixed. Thus, in any case, $T$ leaves a point of $D$ invariant.

5. An example. There exist continua which admit of no continuous transformation into themselves except the identity. We give below an example of a compact acyclic continuous curve in the plane having this property.

Let $A_{1}$ be any arc in the plane of length 1 . Let $A_{2}$ be the sum of $A_{1}$ and two arcs of length $1 / 2^{2}$ having no point in common with each other or with $A_{1}$ except that each has one end point at the midpoint of $A_{1}$. For each integer $n$, let $E_{n-1}$ designate the set of points of $A_{n-1}$ of Menger order greater than two. Let $A_{n}$ be the sum of $A_{n-1}$ and $n$ arcs of length $1 / 2^{n}$ having no point in common with each other or with $A_{n-1}$ except that they all have in common one

* N. E. Rutt, Prime ends and indecomposability, Bulletin of the American Mathematical Society, vol. 41 (1935), pp. 265-273. 
end point which is the midpoint of a component of $A_{n-1}-E_{n-1}$ whose length is not less than the length of any other component of $A_{n-1}-E_{n-1}$. Let $M=\sum_{r=1}^{\infty} A_{r}$. Then $M$ is obviously an acyclic continuous curve. Let $K$ be the set of points of $M$ of Menger order greater than two. From the definition of $M$ it follows that no two points of $K$ are of the same Menger order and that $K$ is everywhere dense in $M$. Let $T$ be any continuous transformation of $M$ into itself. $T$ carries a point of given Menger order into a point of the same Menger order and therefore leaves each point of $K$ fixed. Since $K$ is everywhere dense in $M, T$ leaves each point of $M$ fixed.

It should be noted that $M$ is homeomorphic with a proper subset of itself. E. W. Miller* has given an example of an acyclic continuous curve which is not homeomorphic with any proper subset of itself, but which however does not have the property of the example we have given, since his acyclic continuous curve contains an arc which contains no points of Menger order greater than two. I have not been able to find an example of an acyclic continuous curve in the plane whose only transformation into a subset of itself is the identity.

* E. W. Miller, The Zarankiewicz problem, Bulletin of the American Mathematical Society, vol. 38 (1932), pp. 831-834.

The University of TeXas, Austin, TeXas 